\title{
SIMULTANEOUS DETERMINATION OF DIFFERENT FLAVONOIDS IN HUMAN PLASMA BY A SIMPLE HPLC ASSAY
}

\author{
A. MÜLLER-SEPÚLVEDA ${ }^{1,2 *}$, M. E. LETELIER ${ }^{1,}$ B. SAN MARTIN ${ }^{3}$ \& I. SAAVEDRA-SAAVEDRA² \\ 'Department of Pharmacological and Toxicological Chemistry, Faculty of Chemical and Pharmaceutical Sciences, University of Chile, Sergio Livingstone \\ Pohlhammer (ex-Olivos) 1007, Independencia, Santiago, Chile \\ ${ }^{2}$ Center of Pharmacological and Toxicological Research, Program of Molecular and Clinical Pharmacology, Faculty of Medicine, University of Chile, Carlos \\ Schatebeck 299, Parque Quinta Normal, Santiago, Chile \\ ${ }^{3}$ Laboratory of Veterinary Pharmacology, Faculty of Veterinary Sciences, University of Chile, Santa Rosa 11735, La Pintana, Santiago, Chile
}

\begin{abstract}
A simple HPLC method for simultaneous determination of five flavonoids in human plasma was developed. Following addition of catechin as internal standard (IS), luteolin-7-glucoside, rutin, apigenin-7-glucoside, quercetin and baicalein were isolated from human plasma by liquid-liquid extraction with acetone. The chromatographic separation was achieved in a DAD-HPLC equipment on a reversed-phase C18 column using a gradient mobile phase consisting of $0.1 \%$ acetic acid + methanol: acetonitrile: acetic acid $(90: 10: 1, \mathrm{v} / \mathrm{v})$ and running at a flow rate of $1 \mathrm{~mL} / \mathrm{min}$. The effluent was monitored at a wavelength of $280 \eta \mathrm{m}$, as well as IS was well separated from each other and free from interference from blank plasma and other components in plasma. The calibration curve was constructed by plotting peak area ratio of analytes to IS vs. concentration. The method showed good linearity over range of $5-30 \mu \mathrm{g} / \mathrm{mL}$ for the five standards used ( $\mathrm{r}>0.996)$. The developed method may be used not only to identify the analytes assayed but, simultaneously measure plasma concentrations of these herbal compounds.
\end{abstract}

Keywords:
Flavonoids
Catechin
Rutin
Apigenin-7-glucoside
Luteolin-7-glucoside
Baicalein
Quercetin
HPLC

\section{INTRODUCTION}

Polyphenols are a large family of secondary plant metabolites with recognized antioxidant activity ${ }^{1-4}$. They can be classified in flavonoids, phenolic acids and tannins ${ }^{5}$. The polyphenols used in this study: catechin, rutin, baicalein, quercetin, apigenin-7-glucoside and luteolin-7-glucoside are all flavonoids, the principal group of phenols with antioxidant activities ${ }^{6}$. This antioxidant capacity is due to their ability to reduce free radical formation and to scavenge free radicals, given them their particular pharmacological effect? ${ }^{7}$. That's why it has increased the use of herbal preparations associated with conventional therapy for diseases such as cardiovascular and neurodegenerative; which are specially related with oxidative stress ${ }^{3,8-10}$.

From the pharmacokinetic point of view, the plasma concentration of active principle correlates with the pharmacological effect. But the effects of phytodrugs, who are polyvalent preparations, are due to several compounds ${ }^{11,12}$, which can act synergistically to improve human health ${ }^{11,13,14}$. In this scenario, we think that the determination of a single compound is not representative of the beneficial action of any phytodrug. On the other hand, bioavailability studies from herbal preparations are very diverse $e^{15-18}$ and comparison of pharmacokinetics and pharmacodynamics parameters are difficult to assess. This differences includes determination of one single compound ${ }^{16}$, the dose given to animals or humans are to high compare to normal intake ${ }^{16}$, the administration is not oral but intravenous ${ }^{19}$, or for the determination of this compounds is necessary a very sensitive method like mass cromatography ${ }^{20}$.

HPLC was widely used to separate and analyse flavonoids; however, to our knowledge, there were few literature to report the separation of luteolin7-glucoside, rutin, apigenin-7-glucoside, quercetin and baicalein by HPLC in human plasma. For example, Yang et al. ${ }^{21}$ report the determination of six flavonoids in rat urine and chicken plasma by HPLC with ultraviolet-visible detection, with conditions very similar to ours, but they used chicken plasma not human plasma. Hamed et al. ${ }^{22}$ determine the phenolic profile in samh flour, where they analyse its $\mathrm{MeOH}$ extract in human plasma but by LC-ESI-MS/MS.
Although there are studies that determine different flavonoids, none of them allowed us to determine the concentration in human plasma of selected flavonoids. That's why we develop a simple and easy HPLC method for determination of five flavonoids in human plasma. As mentioned above, simultaneous determination of several antioxidant compounds is a more realistic representation of the compounds that provoke the pharmacological effect suggested. Therefore, the possibility to identify and quantify diverse herbal antioxidant compounds with this method, would allow us to determine the bioavailability of phytodrugs, studies that are being developed in our laboratory.

\section{MATERIAL AND METHODS}

\section{Analytical reagents and chemicals}

Catechin, rutin, quercetin and baicalein were purchased from Sigma Aldrich Chemical Co. (St. Louis, MO, USA). Methanol, acetonitrile and acetic acid were purchased from Merck SA, Chile. Luteolin-7-glucoside and apigenin-7-glucoside were purchased from Fluka. All other reagents used were of PA quality.

\section{Chromatographic conditions}

The liquid chromatographic system used for DAD analysis of extracts was Merck-Hitachi Elite LaChrom equipment consisting of a L-2130 pump, a L-2450 UV diode array detector, and a L-2200 refrigerated autosampler. The analytes were eluted on a Purospher Star RP-18 column ( $150 \mathrm{~mm} \times 4.6 \mathrm{~mm}$ ID, $5 \mu \mathrm{m})$ maintained at room temperature $\left(25^{\circ} \mathrm{C}\right)$. The mobile phase consisted of two solvents, solvent A: $0.01 \%$ acetic acid and solvent B: methanol + acetonitrile + acetic acid, 90:10:1, v/v. The gradient elution programme is shown in Table 1 . The flow rate was $1 \mathrm{~mL} / \mathrm{min}$. The effluent was monitored at a wavelength of $280 \mathrm{\eta m}$ for $76 \mathrm{~min}$ and the volume injected was $40 \mathrm{~mL}$. 
Table 1: Gradient elution programme.

\begin{tabular}{|c|c|c|}
\hline Time (min) & Solution A & Solution B \\
\hline 0 & 90 & 30 \\
\hline 10 & 70 & 30 \\
\hline 15 & 70 & 40 \\
\hline 25 & 60 & 50 \\
\hline 40 & 50 & 100 \\
\hline 50 & 0 & 100 \\
\hline 60 & 0 & 100 \\
\hline 70 & 0 & 10 \\
\hline 70.1 & 90 & 10 \\
\hline
\end{tabular}

Values of solution A and B represent the percentage of each solvent to complete $100 \%$.

\section{Preparation of standards}

A mixture stock solution of luteolin-7-glucoside, rutin, apigenin-7glucoside, quercetin and baicalein $(1 \mathrm{mg} / \mathrm{mL})$ were prepared in methanol. The internal standard solution containing $1 \mathrm{mg} / \mathrm{mL}$ of catechin was prepared in the same solvent. These solutions were stored at $-20^{\circ} \mathrm{C}$. The working standard solutions containing $5,10,15,20,25$ and $30 \mu \mathrm{g} / \mathrm{mL}$ were freshly prepared by serially diluting the above mixture solution of standards with methanol. The range of concentrations used is small because the concentration of flavonoids in herbal extracts is very little, gathering our efforts to determine the lowest possible concentrations with the laboratory equipment and conditions.

\section{Human Plasma}

The Clinical Laboratory of Hospital San Juan de Dios donated human plasma to the Laboratory of Pharmacological and Toxicological Research of the University of Chile, with all ethical considerations necessary to use plasma samples for the study.

\section{Plasma sample preparation}

A $1000 \mu \mathrm{L}$ of human plasma sample was transferred into an Eppendorf tube containing $50 \mu \mathrm{L}$ of IS at $0.1 \mathrm{mg} / \mathrm{mL}, 50 \mu \mathrm{L}$ of acetic acid and $500 \mu \mathrm{L}$ of methanol. The resultant mixture was vortex for $5 \mathrm{~min}$, sonicates for $10 \mathrm{~min}$ and incubated for $30 \mathrm{~min}$ at $37^{\circ} \mathrm{C}$ with constant agitation. Then the mixture was extracted twice with $1000 \mu \mathrm{L}$ of acetone each by vortex mixing for $5 \mathrm{~min}$, sonicated for $10 \mathrm{~min}$ and centrifuged at $3000 \times \mathrm{g}$ for $10 \mathrm{~min}$ at $4^{\circ} \mathrm{C}$. The upper organic phase was transferred into another Eppendorf tube and evaporated to dryness under a gentle $\mathrm{N} 2$ stream at $50^{\circ} \mathrm{C}$ in a water bath. The residue was reconstituted in $300 \mu \mathrm{L}$ of mobile phase. Then $40 \mu \mathrm{L}$ of supernatant was injected onto chromatograph for analysis (Figure 1).

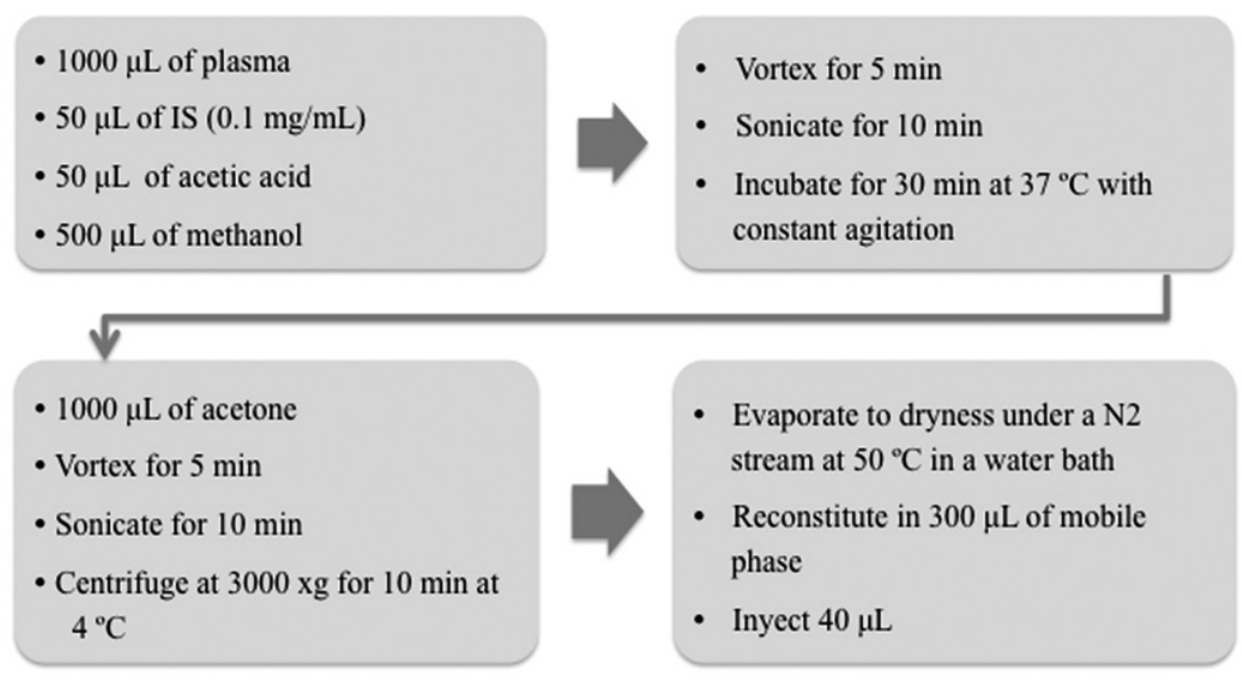

Figure 1: Plasma sample preparation.

\section{Linearity of HPLC system and its sensibility}

Linear regression was obtained by plotting the peak-height ratio of a series of dilutions for each phenolic compound against the known concentration of the analytes in the stock standard solution. The regression lines, expressed as correlation coefficients, were linear and close to one $(r>0.996)$ for each parameter in the studied range, as shown in Table 2. Sensitivity (defined as the lowest measurable concentration of a compound in the sample) was estimated as the concentration that generated a peak with a height that was at least threetimes higher than the baseline noise range.

\section{Validation of methodology}

This HPLC assay was validated according to the Guideline on Bioanalytical Method Validation ${ }^{18}$. The parameters evaluated were: selectivity, linearity, carry-over, accuracy and precision. Apigenin-7-glycoside and rutin, polyphenol standards with different chemical structure, were used to validate the HPLC method implemented. Standard curves of these compounds are shown in Figure 4.

\section{RESULTS AND DISCUSSION}

\section{Internal Standard}

An appropriate internal standard is needed for accurate quantification of analytes. We tested different flavonoids standards to select the appropriate internal standard which gives the suitable retention time and no interference from endogenous matrix. Catechin was finally selected as the optimal internal standard because it had a retention time separated from others flavonoids and good resolution under the chromatographic conditions established (Figure 2).

\section{Detection wavelength}

The maximal absorbance wavelengths $\left(\lambda_{\max }\right)$ of five flavonoids were analysed by scanning between 200 and $500 \mathrm{~nm}$ on the Merck-Hitachi Elite LaChrom L-2450 UV diode array detector, in order to obtain the optimal detection wavelength for the chromatography separation. The results of UV spectra showed that the absorbance peaks of five flavonoids were different, but they all had the best absorbance peaks at $280 \eta \mathrm{m}$. Therefore, the UV-vis detector was set at the wavelength of $280 \mathrm{\eta m}$ in this method. 


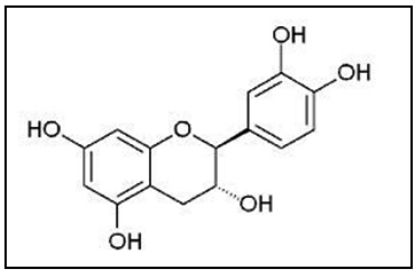

Catechin

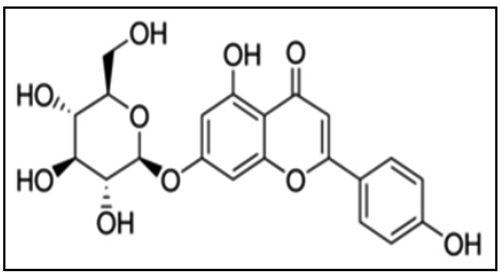

Luteolin-7-glucoside

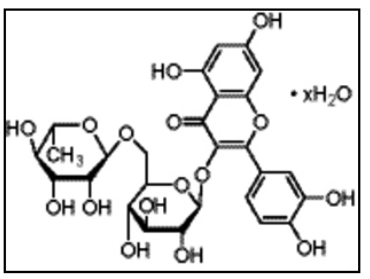

Rutin

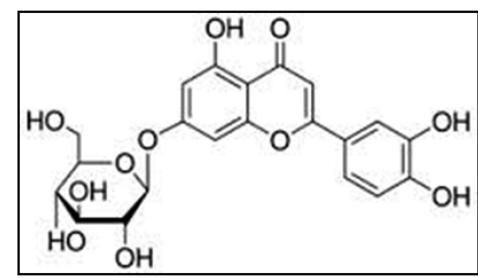

Apigenin-7-glucoside

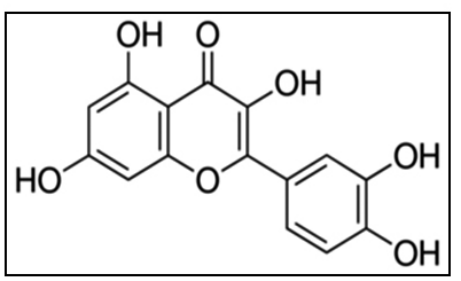

Quercetin<smiles>O=c1cc(-c2ccccc2)oc2cc(O)c(O)c(O)c12</smiles>

Baicalein

Figure 2. Chemical structure of flavonoid standard used.

\section{Analytical method}

Determination of HPLC separation procedure for quantitative analysis was difficult to achieve due to the highly complex chromatographic profile, which showed five flavonoids. Different mobile phases conditions were tested to acquire the best analytical results. Acetone was found to provide the highest recoveries compared with others such as methanol, acetonitrile, ethanol, diethyl ether and ethyl acetate. Furthermore, two-extraction with acetone give better results than once-extraction. Pre-treatment of plasma samples with acetic acid for protein precipitation was associated with best recoveries than the use of hydrochloric acid. The effect of mobile phase on retention and resolution of the five flavonoids were investigated. After proving isocratic and gradient mobile phases, the mobile phase consisting of 2 solvents; solvent A: $0.01 \%$ acetic acid and solvent B: methanol + acetonitrile + acetic acid 90:10:1, v/v was chosen as it gave a shortest retention time, better shapes of peaks, and good resolution between catechin, luteolin-7-glucoside, rutin, apigenin-7-glucoside, quercetin, baicalein and matrix. Figure 3 show the chromatogram of blank plasma with IS and Figure 4 show the chromatograph of all flavonoids tested.

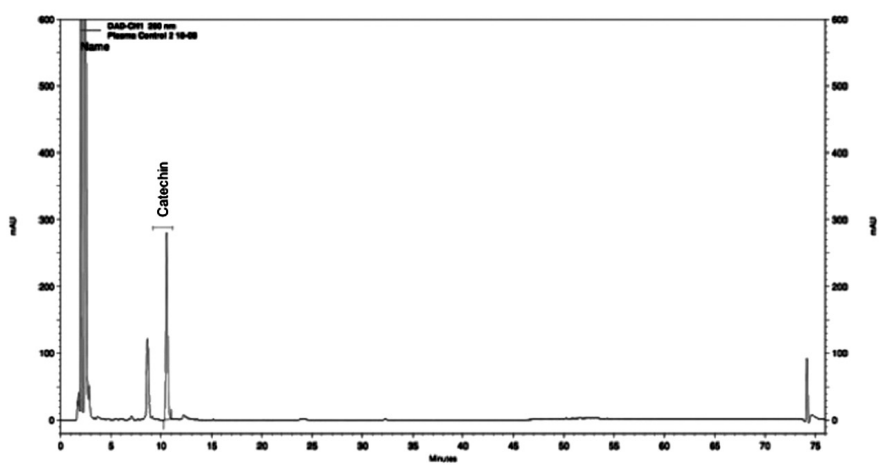

Figure 3. Chromatogram of human plasma in the presence of IS determined at $280 \eta \mathrm{m}$. Catechin concentration was $175 \mu \mathrm{g} / \mathrm{ml}$.

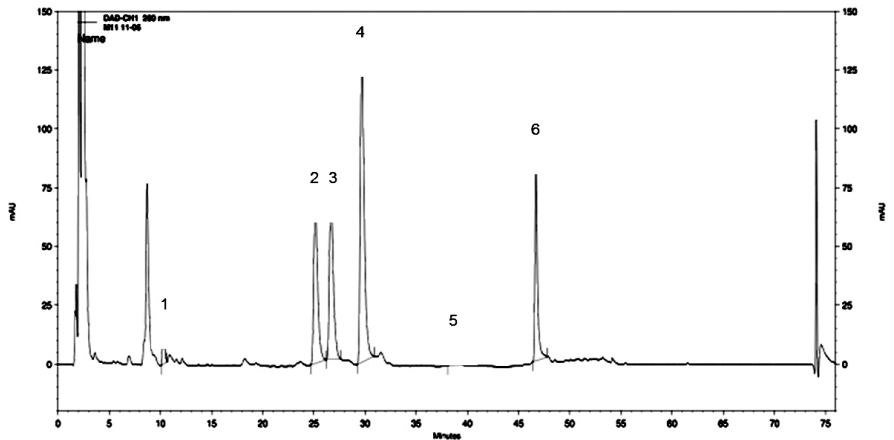

Figure 4. Chromatogram of the standard mixture solubilized in human plasma and determined at $280 \eta \mathrm{m}$. 1: catechin; 2: luteolin-7-glucoside; 3: rutin; 4: apigenin-7-glucoside; 5: quercetin; 6: baicalein. The concentration of the standard in the mixture was $100 \mu \mathrm{g} / \mathrm{mL}$, except for the internal standard that was $17.5 \mu \mathrm{g} / \mathrm{mL}$.

Figure 4 showed that luteolin-7-glucoside, rutin, apigenin-7-glucoside, quercetin, baicalein and IS were separated well from each other with retention time (TR) of 24.7, 26.2, 29.3, 38.1, 46.7 and $6.0 \mathrm{~min}$, respectively. There was no interference from blank plasma and from metabolites. Moreover, other components or impurities of plasma did not interfere with analytes and IS.

\section{Calibration curves}

Plasma calibration curves of each analytes were established by injecting six different concentrations. The results found to be linear over studied range of 5-30 $\mu \mathrm{g} / \mathrm{mL}$ for all flavonoids. Figure 5 show the calibration curves and Table 2 , the retention time and the correlation coefficients of the calibration curves. 

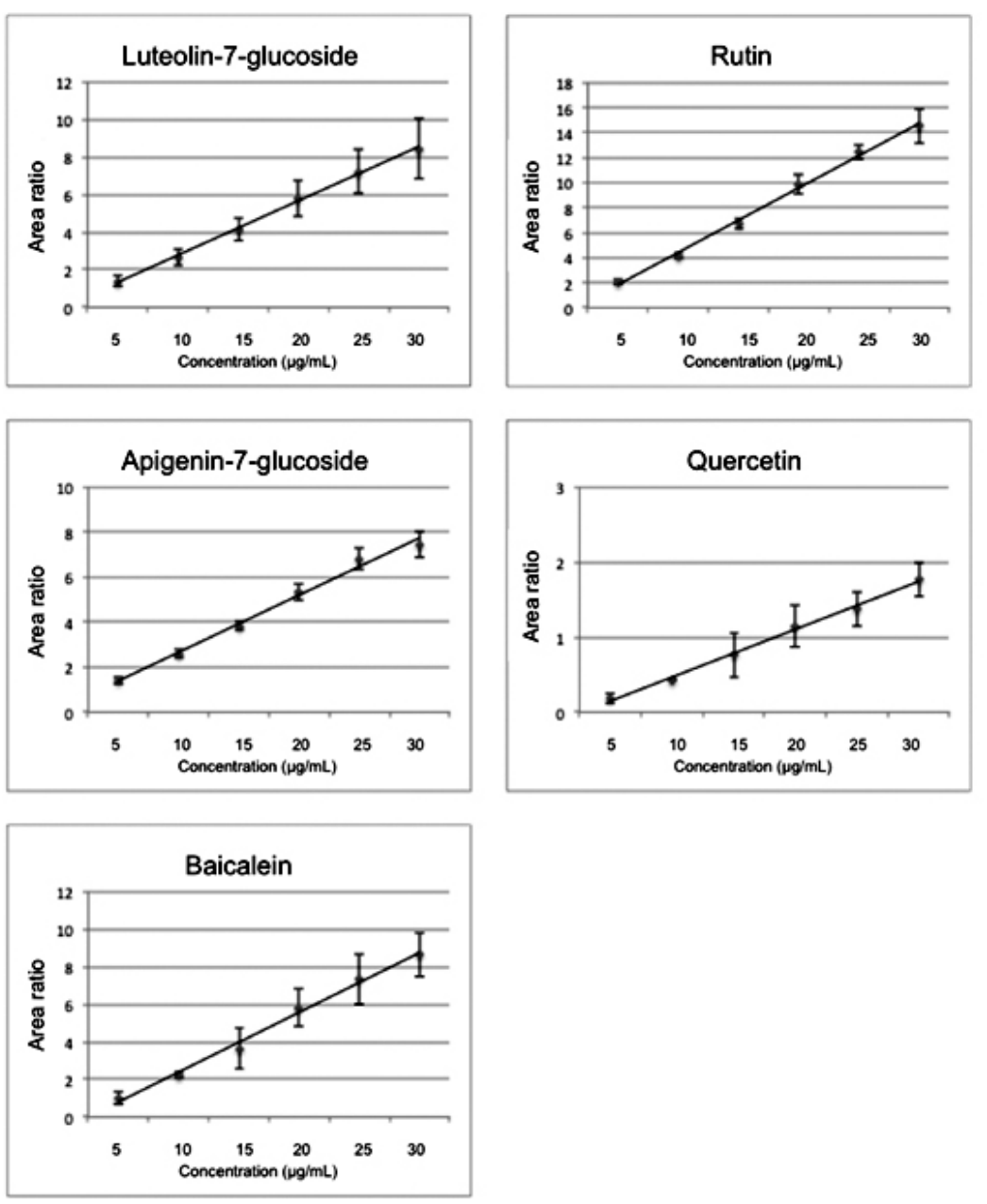

Figure 4. Calibration curves of standards used. Each point on the lines represents the average of at least 5 independent experiments \pm SD.

Table 2. Retention time and correlation coefficients of the flavonoid used.

\begin{tabular}{|c|c|c|}
\hline Standard & Retention time (min) & r \\
\hline Luteolin-7-glucoside & 25.320 & 0.999 \\
\hline Apigenin-7-glucosid & 26.707 & 0.996 \\
\hline Rutin & 29.740 & 0.995 \\
\hline Quercetin & 38.660 & 0.998 \\
\hline Baicalein & 46.727 & 0.997 \\
\hline
\end{tabular}

Determination of herbal analyte concentration

Calibration curves allow to quantify the flavonoids concentration in plasma, through the following equation: $\mathrm{y}=\mathrm{mx}+\mathrm{n}$, where: $\mathrm{y}=$ peak area ratio of analyte/IS

$\mathrm{m}=$ slope of straight line

$\mathrm{n}=$ intercept of straight line

$\mathrm{x}=[$ plasma analyte $]$ expressed as $\mu \mathrm{g} / \mathrm{mL}$

\section{Sensibility}

The property of the analytical method for detecting an analyte, even in the presence of other components such as impurities, degradation products or components of the formulation is called selectivity. Six different sources of human plasma were used to determine if this matrix exhibited interference with the analytes under study. Comparison between the area ratio of plasma peaks and internal standard, showed no interference in the retention times of the standards used.

\section{Carry-over}

Carry-over corresponds to interference that can generate from analytes retained on the column and/or previous runs. To corroborate this, mobile phase was injected in absence of analytes. The analysis of the chromatograms showed no entrainment compounds. To verify this parameter routinely, this procedure is carried out every 6 runs samples.

\section{Accuracy and Precision}

Accuracy is the closeness of the results of measurements to the true 
value. It is calculated according to the relative error. Precision is the degree of dispersion between the results of a series of measurements corresponding to a same biological sample. It is expressed as standard deviation and coefficient of variation (CV). According to the $\mathrm{FDA}^{23}$, for each level of concentration, relative error and CV should be less than $15 \%$, except for the lowest concentration, where $20 \%$ is the smaller percentage accepted. Table 3 and 4 show the accuracy and precision of apigenin-7-glucoside and rutin. Both flavonoids show a relative error smaller than $8 \%$ and a CV smaller than $11 \%$, validating the accuracy and precision of the analytical method implemented.

Table 3. Accuracy and Precision of apigenin-7-glucoside.

\begin{tabular}{|c|c|c|c|c|c|c|}
\hline $\begin{array}{c}\text { Apigenin-7- } \\
\text { glucoside Real } \\
{[\mu \mathrm{g} / \mathrm{mL}]}\end{array}$ & RA & $\begin{array}{c}\text { Apigenin-7-glucoside } \\
\text { Experimental } \\
{[\mu \mathrm{g} / \mathrm{mL}]}\end{array}$ & $\begin{array}{c}\text { [Real]/ } \\
{[\text { Experimental] }}\end{array}$ & $\begin{array}{c}\text { Accuracy } \\
\mathbf{( \% )}\end{array}$ & $\begin{array}{c}\text { Relative } \\
\text { Error } \\
\mathbf{( \% )}\end{array}$ & $\begin{array}{c}\text { CV } \\
\mathbf{( \% )}\end{array}$ \\
\hline 5 & 1.39 & $5.01 \pm 0.15$ & 1.01 & 100.19 & 0.19 & 10.99 \\
\hline 10 & 2.59 & $9.71 \pm 0.17$ & 0.97 & 97.08 & 2.92 & 6.60 \\
\hline 15 & 3.84 & $14.63 \pm 0.19$ & 0.98 & 97.55 & 2.45 & 4.85 \\
\hline 20 & 5.32 & $20.45 \pm 0.35$ & 1.02 & 102.24 & 2.24 & 6.55 \\
\hline 25 & 6,82 & $26.33 \pm 0.48$ & 1.05 & 105.31 & 5.31 & 7.04 \\
\hline 30 & 7.46 & $28.88 \pm 0.58$ & 0.96 & 96.25 & 3.75 & 7.76 \\
\hline
\end{tabular}

EEach experimental concentration value represents the average of at least 5 independent experiments $\pm \mathrm{SD}$. RA: area ratio between the peaks of the flavonoid used with respect to the internal standard, CV: coefficient of variation.

Table 4. Accuracy and Precision of rutin.

\begin{tabular}{|c|c|c|c|c|c|c|}
\hline $\begin{array}{c}\text { Rutin } \\
\text { Real } \\
{[\mu \mathrm{g} / \mathrm{mL}]}\end{array}$ & RA & $\begin{array}{c}\text { Rutin } \\
\text { Experimental } \\
{[\mu \mathrm{g} / \mathrm{mL}]}\end{array}$ & $\begin{array}{c}{[\text { Real]/ }} \\
{[\text { Experimental] }}\end{array}$ & $\begin{array}{c}\text { Accuracy } \\
\mathbf{( \% )}\end{array}$ & $\begin{array}{c}\text { Relative } \\
\text { Error } \\
\mathbf{( \% )}\end{array}$ & $\begin{array}{c}\text { CV } \\
\mathbf{( \% )}\end{array}$ \\
\hline 5 & 2.03 & $5.38 \pm 0.21$ & 1.08 & 107.67 & 7.67 & 10.32 \\
\hline 10 & 4.11 & $9.40 \pm 0.25$ & 0.94 & 94.01 & 5.99 & 6.10 \\
\hline 15 & 6.71 & $14.45 \pm 0.39$ & 0.96 & 96.30 & 3.70 & 5.76 \\
\hline 20 & 9.87 & $20.57 \pm 0.77$ & 1.03 & 102.82 & 2.82 & 7.81 \\
\hline 25 & 12.90 & $26.44 \pm 1.18$ & 1.06 & 105.75 & 5.75 & 9.18 \\
\hline 30 & 14.17 & $28.89 \pm 1.43$ & 0.96 & 96.29 & 3.71 & 10.09 \\
\hline
\end{tabular}

Each experimental concentration value represents the average of at least 5 independent experiments \pm SD. RA: area ratio between the peaks of the flavonoid used with respect to the internal standard. CV: coefficient of variation.

\section{CONCLUSION}

The present study describes a HPLC method for simultaneous determination of five different standards of flavonoids in human plasma, which was validated to be simple, specific, precise and thus, completely suitable for pharmacokinetic study of herbal preparations. There are many studies that determine de concentration of flavonoids or others antioxidant compounds in leaves, fruits, flowers or different parts of plants using mainly HPLC methodo$\operatorname{logies}{ }^{24-29}$. But the determinations of flavonoids in biological fluids such as plasma is much more complicated and only a limited number of studies are in scientific literature ${ }^{16,30}$. When trying to determine these compounds in plasma, there are different compounds like proteins vitamins, cells, detritus, waste products, etc. that can interfere with the reading, so today these determination are mainly performed in HPLC coupled to mass spectrometry. This equipment has a high sensitivity, but is expensive and in Chile only a few laboratories can have access to them.

When the methodology used to quantify flavonoids in plasma is HPLC DAD, most of the times only one or two compounds can be determine, because the diversity of chemical structures of antioxidant molecules make difficult to separate them from biological matrix. Another problem that can be presented is that concentration achieves in plasma is very low because the bioavailability of polyphenols can vary from $80 \%$ to $15 \%{ }^{31}$, so the recoveries may also be low. This method allows determining the presence and concentration of herbal antioxidants in plasma. We determine not only one, but five different flavonoids, which give us a more realistic point of view of what is happening in vivo in the human body. The method employed simple sample preparation for human plasma samples, and it is easily adaptable in many laboratories using commonly available HPLC equipment. We hope this simple method can helps increase the knowledge of phytodrugs to improve health of human and animals, by allowing the determination of bioavailability by the implementation of pharmacokinetic studies of medicinal plants.

\section{ACKNOWLEDGMENTS}

We are grateful to Obens Laboratory and Laboratory of Veterinary Pharmacology, Faculty of Veterinary Sciences, University of Chile (Dr. Betty San Martín).

\section{REFERENCES}

1. Quideau S, Deffieux D, Douat-Casassus C, Pouységu L. Plant Polyphenols: Chemical Properties, Biological Activities, and Synthesis. Angewandte Chemie International Edition. 586-621, (2011).

2. Rah DK, Han D-W, Baek HS, Hyon S-H, Park J-C. Prevention of reactive oxygen species-induced oxidative stress in human microvascular endothelial cells by green tea polyphenol. Toxicol. Lett.155: 269-75, (2005).

3. Sies H. Polyphenols and health : Update and perspectives. Arch Biochem Biophys. 501:2-5, (2010). 
4. Tapiero H, Tew KD, Ba GN, Mathé G. Polyphenols: do they play a role in the prevention of human pathologies? Biomed Pharmacother. 56(4): 200-207, (2002).

5. Martins S, Mussatto SI, Martínez-Avila G, Montañez-Saenz J, Aguilar CN, Teixeira JA. Bioactive phenolic compounds: Production and extraction by solid-state fermentation. A review. Biotechnol Adv. 29(3): 365-373, (2011).

6. Scalbert A, Williamson G. Dietary Intake and Bioavailability of Polyphenols. J Nutr. 130: 2073-2085, (2000).

7. Quideau S, Deffieux D, Douat-Casassus C, Pouységu L. Plant Polyphenols: Chemical Properties, Biological Activities, and Synthesis. In: Angewandte Chemie International Edition.Vol 50: 586-621, (2011).

8. Rahman I, Biswas SK, Kirkham PA. Regulation of inflammation and redox signaling by dietary polyphenols. Biochem Pharmacol. 72(11): 1439-1452, (2006;).

9. Reuter S, Gupta SC, Chaturvedi MM, Aggarwal BB. Oxidative stress, inflammation, and cancer: How are they linked? Free Radic Biol Med. 49(11): 1603-1616, (2010).

10. Del Rio D, Costa LG, Lean MEJ, Crozier A. Polyphenols and health: what compounds are involved? Nutr Metab Cardiovasc Dis. 20(1): 1-6, (2010).

11. Pignatelli P, Ghiselli A, Buchetti B, et al. Polyphenols synergistically inhibit oxidative stress in subjects given red and white wine. Atherosclerosis. 188(1): 77-83, (2006).

12. Wagner H. Synergy research: Approaching a new generation of phytopharmaceuticals. Fitoterapia. 82:34-37, (2011).

13. Ulrich-Merzenich G, Panek D, Zeitler H, Vetter H, Wagner H. Drug development from natural products: exploiting synergistic effects. Indian J Exp Biol. 48(3): 208-219, (2010).

14. Tallarida RJ. Drug synergism: its detection and applications. J Pharmacol Exp Ther. 298(3): 865-872, (2001).

15. Williamson G, Manach C. Bioavailability and bioefficacy of polyphenols in humans. II. Review of 93 intervention studies. Am J Clin Nutr. 81(1 Suppl): 243S - 255S, (2005).

16. Bell JR, Donovan JL, Wong R, et al. (+)-Catechin in human plasma after ingestion of a single serving of reconstituted red wine. Am J Clin Nutr. 71(1): 103-108, (2000).

17. Maiani G, Serafini M, Salucci M, Azzini E, Ferro-Luzzi A. Application of a new high-performance liquid chromatographic method for measuring select polyphenols in human plasma. J Chromatogr B. 692(2): 311-317, (1997).

18. Abrahamse SL, Kloots WJ, van Amelsvoort JMM. Absorption, distribution, and secretion of epicatechin and quercetin in the rat. Nutr Res. 25(3): 305-317, (2005).

19. Fu T, Liang J, Han G, Lv L, Li N. Simultaneous determination of the major active components of tea polyphenols in rat plasma by a simple and specific HPLC assay. J Chromatogr B Analyt Technol Biomed Life Sci. 875(2): 363-367, (2008).

20. Yang Z, Zhu W, Gao S, et al. Simultaneous determination of genistein and its four phase II metabolites in blood by a sensitive and robust UPLC-MS/ MS method: Application to an oral bioavailability study of genistein in mice. J Pharm Biomed Anal. 53(1): 81-89, (2010).

21. Yang GJ, Liu $\mathrm{P}, \mathrm{Qu} \mathrm{XL}$, et al. The simultaneous separation and determination of six flavonoids and troxerutin in rat urine and chicken plasma by reversed-phase high-performance liquid chromatography with ultraviolet-visible detection. J Chromatogr B Anal Technol Biomed Life Sci. 856(1-2): 222-228, (2007).

22. Hamed AI, Said R Ben, Kontek B, et al. LC-ESI-MS/MS profile of phenolic and glucosinolate compounds in samh flour (Mesembryanthemum forsskalei Hochst. ex Boiss) and the inhibition of oxidative stress by these compounds in human plasma. Food Res Int. 85:282-290, (2016).

23. European Medicines Agency. Guideline on Bioanalytical Method Validation. (2012).

24. Marston A. Role of advances in chromatographic techniques in phytochemistry. Phytochemistry. 68(22-24): 2786-2798, (2007).

25. Simirgiotis MJ, Schmeda-Hirschmann G. Determination of phenolic composition and antioxidant activity in fruits, rhizomes and leaves of the white strawberry (Fragaria chiloensis spp. chiloensis form chiloensis) using HPLC-DAD-ESI-MS and free radical quenching techniques. J Food Compos Anal. 23(6): 545-553, (2010).

26. Vasco C, Riihinen K, Ruales J, Kamal-Eldin A. Phenolic compounds in Rosaceae fruits from Ecuador. J Agric Food Chem. 57(4): 1204-1212, (2009).

27. Abad-García B, Berrueta L a, Garmón-Lobato S, Gallo B, Vicente F. A general analytical strategy for the characterization of phenolic compounds in fruit juices by high-performance liquid chromatography with diode array detection coupled to electrospray ionization and triple quadrupole mass spectrometry. J Chromatogr A. 1216(28): 5398-5415, (2009).

28. Sakakibara H, Honda Y, Nakagawa S, Ashida H, Kanazawa K. Simultaneous determination of all polyphenols in vegetables, fruits, and teas. J Agric Food Chem. 51(3): 571-581, (2003).

29. Zhang W, Han F, He J, Duan C. HPLC-DAD-ESI-MS/MS Analysis and Antioxidant Activities of Nonanthocyanin Phenolics in Mulberry (Morus alba L.). J Food Sci. 73(6): C512-C518, (2008).

30. Funes L, Fernández-Arroyo S, Laporta O, et al. Correlation between plasma antioxidant capacity and verbascoside levels in rats after oral administration of lemon verbena extract. Food Chem. 117(4): 589-598, (2009).

31. D'ArchivioM,FilesiC,VarìR,ScazzocchioB,MasellaR.Bioavailabilityofthe polyphenols: statusand controversies.IntJMolSci.11(4):1321-1342,(2010). 